\title{
Special Issue Editorial: Computational Intelligence and Applications
}

\author{
Zhigang Zeng • Haibo He
}

Received: 5 December 2012/ Accepted: 3 February 2013/Published online: 17 February 2013

(C) Springer Science+Business Media New York 2013

This special issue includes seven highly selected papers from the Fourth International Workshop on Advanced Computational Intelligence (IWACI 2011), held from October 19 to 21, 2011, in Wuhan, China. The IWACI 2011 was a great success and provided a high-level international forum for scientists and engineers to present the latest research in the filed of computational intelligence and applications. To highlight the success of this conference, we edited this special issue for the Cognitive Computation. We chose seven papers from 412 papers submitted to the IWACI'11, as these papers reflect the high quality of the presentations at the conference while capturing the spirit of our theme, "Computational Intelligence and Applications," for this special issue.

Over the past decades, we have witnessed tremendous efforts and developments from all aspects of computational intelligence research, ranging from theoretical foundations, principles, algorithms, to practical applications in different domains. To reflect a flavor of such recent research activities in the community, we carefully selected these seven papers for this special issue, with a focus on different computational intelligence techniques and real-world applications.

\footnotetext{
Z. Zeng

Department of Control Science and Engineering,

Huazhong University of Science and Technology,

Wuhan 430074, China

e-mail: zgzeng@gmail.com

H. He $(\square)$

Department of Electrical, Computer, and Biomedical

Engineering, University of Rhode Island,

Kingston, RI 02881, USA

e-mail: he@ele.uri.edu
}

The first group of papers is focused on the computational intelligence techniques for image processing. As computer vision and image processing has become a critical issue for many of today's data-intensive applications, ranging from civilian applications such as video processing to surveillance and security-related applications, we selected three papers on this topic. The paper by Zhao et al. discussed an important method for non-blind image de-blurring from a single image. In order to overcome the limitations of the traditional maximum a posteriori (MAP) estimation for nonblind image de-blurring, the authors proposed a Bayesian minimum mean squared error (MMSE) estimation to perform de-blurring. This method is based on an effective statistical framework to model prior knowledge of natural images with an efficient Gibbs sampling algorithm. Various simulations and comparative studies have been developed to demonstrate the effectiveness of this approach. In the paper by Ding et al., a method based on neural network and selforganizing map (SOM) was proposed to connect a sequence of images acquired by a rotating camera. This is a very interesting problem with broader practical applications. A detailed algorithm with a system-level implementation has been presented in this paper. Image classification was studied in the paper by Bian et al. Specifically, the authors presented a method based on modified k-means support vector machines (SVMs) with hybrid sampling method for image classification. This method can reduce convex hulls of clustering structure as well as the overtraining caused by active sampling approaches. Simulation results based on three challenging remote sensing images with comparative study of random sampling (RS) and margin sampling (MS) demonstrated the effectiveness of this approach.

The second group includes two theoretically focused papers. In the paper by Feng et al., an interesting problem of stochastic suppression and stabilization of nonlinear 
system has been studied. Specifically, the authors presented the theoretical study for nonlinear system with Markovian switching, whose coefficients satisfy the one-sided polynomial growth condition and the local Lipschitz condition. This paper demonstrated that given an unstable differential system under certain conditions, the system can be suppressed and stabilized by noises. The paper further proved that the appropriate conditions may guarantee that stochastic system exists as a unique global solution, although the corresponding deterministic may explode in a finite time. In another theoretically oriented paper, Liu et al. studied the Hopf bifurcation for a modified Leslie-Gower predator-prey system, in which the harvest effort was analyzed from an economic perspective. New criteria for the Hopf bifurcation and a numerical example have been presented to demonstrate the effectiveness of this research.

As optimization plays an important role in many decision-making processes, we also selected one paper on multimodal function optimization based on particle swarm optimization (PSO). Specifically, Tang et al. proposed to use multiple swarms for parallel search, with the integration of artificial repulsive potential field on local search space to prevent multiple swarms from converging to the same area. Various simulation results have been presented to demonstrate the effectiveness of this method.
We also selected one paper directly related to neural network applications. Specifically, Chen et al. presented a method by combining genetic algorithm and simulated annealing algorithm to optimize the weights and biases of a neural network for landslide prediction application. Based on the data collected from the Three Gorges reservoir, the authors demonstrated the effectiveness of this method in a critical real-world application.

In summary, we hope the selected papers in this special issue can demonstrate some of the latest theoretical research and applications presented at the IWACI'11, and will motivate future research directions in this field.

In closing, we would like to express our gratitude to many reviewers who helped us in the peer-review process for this special issue. Their expertise guaranteed the high qualify of the selected papers. We would also like to thank Dr. Amir Hussain, the Editor-in-Chief of Cognitive Computation, for his strong support in bringing the special issue to the audience. Finally, we would like to thank all the authors for their hard work to make this special issue possible.

We hope you will enjoy reading this special issue.

Zhigang Zeng and Haibo He

Guest Editors 